\title{
Diabatic description of charmoniumlike mesons. II. Mass corrections and strong decay widths
}

\author{
R. Bruschini $\oplus^{1, *}$ and P. González $\oplus^{1,2, \dagger}$ \\ ${ }^{1}$ Unidad Teórica, Instituto de Física Corpuscular (Universidad de Valencia-CSIC), \\ E-46980 Paterna (Valencia), Spain \\ ${ }^{2}$ Departamento de Física Teórica, Universidad de Valencia, E-46100 Burjassot (Valencia), Spain
}

(Received 13 January 2021; accepted 10 March 2021; published 13 April 2021)

\begin{abstract}
From a diabatic bound state approach to $J^{P C}=1^{--}$and $(0,1,2)^{++}$charmoniumlike resonances below 4.1 GeV, formulated in terms of $c \bar{c}$ and closed meson-meson channels, we calculate mass shifts and widths due to open meson-meson channels. This calculation does not involve any new free parameter, so comparison of our predictions with existing data provides a direct test of our approach. Further mass corrections are also estimated and good agreement with the measured masses comes out. As for the calculated widths, overall reasonable, they point out to the need of some refinement of our current bound state approximation for an accurate description of data. These results give additional support to the diabatic approach in QCD as an adequate framework for a complete unified description of conventional and unconventional charmoniumlike resonances. In this respect, the experimental discovery of a predicted $2^{++}$ resonance with a mass around $4 \mathrm{GeV}$ would be of special relevance.
\end{abstract}

DOI: 10.1103/PhysRevD.103.074009

\section{INTRODUCTION}

A current challenge in hadron physics is to achieve a QCD based description of (unconventional) quarkoniumlike mesons such as $\chi_{c 1}(3872), X(3915)$, and others [1] discovered in the past two decades, whose properties do not correspond to a very dominant conventional heavy quark $(Q)$-heavy antiquark $(\bar{Q})$ meson structure.

Although there are nowadays compelling experimental indications of the presence of additional open-flavor mesonmeson components in some of these unconventional states, the QCD theoretical implementation of such components, together with the $Q \bar{Q}$ ones for a complete quarkoniumlike description, presents some difficulties (for a comprehensive review of the experimental and theoretical situation, see [2-14] and references therein). Just recently, noticeable progress in this direction, based on lattice QCD results, has been reported. More concretely, unquenched lattice QCD calculations of the energies for static $Q$ and $\bar{Q}$ sources, when the $Q \bar{Q}$ configuration mixes with one or two open-flavor meson-meson configurations, have been carried out $[15,16]$. These static energies can be directly related to the potential

\footnotetext{
*oberto.bruschini@ific.uv.es

pedro.gonzalez@uv.es
}

Published by the American Physical Society under the terms of the Creative Commons Attribution 4.0 International license. Further distribution of this work must maintain attribution to the author(s) and the published article's title, journal citation, and DOI. Funded by SCOAP ${ }^{3}$. matrix entering in a multichannel Schrödinger equation for the $Q \bar{Q}$ and meson-meson components of a quarkoniumlike meson. This relation has been used to explore the bottomoniumlike spectrum when only one (average) meson-meson channel is taken into account [17]. A more general and systematic formulation of this relation for any number of meson-meson channels has been recently proposed [18]. This formulation translates the diabatic approach used in molecular physics to tackle the electronic configuration mixing problem [19] to the study of quarkoniumlike systems.

The direct diabatic correspondence between lattice QCD calculations and the potential matrix entering the Schrödinger equation constitutes a substantial improvement over existing descriptions of quarkoniumlike mesons where the interaction between $Q \bar{Q}$ and meson-meson degrees of freedom has no clear connection with QCD.

It is worth emphasizing that the diabatic approach goes beyond the (single-channel) Born-Oppenheimer approximation in QCD developed in [20] and applied in [21] to the description of quarkonium and quarkonium hybrids. Indeed, the diabatic approach reduces to the Born-Oppenheimer approximation for conventional quarkonium $Q \bar{Q}$ for energies far below the lowest open-flavor meson-meson threshold, but unlike the Born-Oppenheimer approximation it maintains its validity for energies close below or above that threshold. We refer to the previous paper of this series [18] for a more detailed explanation about the advantages of the diabatic approach over the Born-Oppenheimer approximation.

Therefore, the so-called diabatic approach in QCD provides an appropriate framework for a unified and 
complete nonperturbative description of conventional quarkonium and quarkoniumlike mesons made of $Q \bar{Q}$ and meson-meson components.

In the first practical application of the diabatic formalism to the description of charmoniumlike mesons we have considered them stable against decays into open-flavor meson-meson channels, neglecting the fact that states above any possible meson-meson threshold with the same quantum numbers are unstable. This approximation has been implemented by solving the multichannel Schrödinger equation taking into account that a bound state solution with a correct asymptotic behavior requires that meson-meson components with thresholds below the mass of the bound state be neglected. This description, which has been applied to isoscalar $I=0$ states with $J^{P C}=1^{--}$and $(0,1,2)^{++}$below $4.1 \mathrm{GeV}$, is expected to be well suited for narrow states as the ones below the first absolute meson-meson threshold $D \bar{D}$, or the first excited $1^{++}$state, which is assigned to $\chi_{c 1}(3872)$, for which there is no coupled lower threshold. For the other states the treatment may also be suited if mass corrections due to the neglected meson-meson components and widths can be properly incorporated.

In this article we go a step further in the diabatic description of quarkoniumlike mesons by evaluating, from the calculated bound state solutions, the contribution of the neglected meson-meson components to their masses and decay widths. Then, a more detailed comparison with data may be done. In this regard, one should always keep in mind that there might still exist experimental quarkoniumlike resonances escaping from the bound state approximation we follow.

The contents of the article are organized as follows. In Sec. II a brief resume of the diabatic bound state description of quarkoniumlike mesons is presented. In Sec. III we detail the framework for the calculation of mass corrections and widths to open-flavor meson-meson channels. The results obtained for charmoniumlike systems are listed and discussed in Sec. IV. Finally, in Sec. V we summarize our main results and conclusions.

\section{DIABATIC BOUND STATE DESCRIPTION OF QUARKONIUMLIKE MESONS}

The diabatic approach in QCD has been detailed in [18]. Quarkoniumlike mesons, characterized by quantum numbers $J^{P C}$, are assigned to solutions of the multichannel Schrödinger equation,

$$
(\mathrm{K}+\mathrm{V}(r)) \Psi(\boldsymbol{r})=E \Psi(\boldsymbol{r}),
$$

where $\Psi(\boldsymbol{r})$ is a column vector notation for the wave function,

$$
\Psi(\boldsymbol{r})=\left(\begin{array}{c}
\psi_{Q \bar{Q}}(\boldsymbol{r}) \\
\psi_{M \bar{M}}^{(1)}(\boldsymbol{r}) \\
\vdots \\
\psi_{M \bar{M}}^{(N)}(\boldsymbol{r})
\end{array}\right)
$$

with $\psi_{M \bar{M}}^{(i)}(\boldsymbol{r}), i=1,2 \ldots$ standing for the $i$ th meson-meson component.

$\mathrm{K}$ is the kinetic energy matrix

$$
\mathrm{K}=\left(\begin{array}{cccc}
-\frac{1}{2 \mu_{Q \bar{Q}}} \nabla^{2} & & & \\
& -\frac{1}{2 \mu_{M \bar{M}}^{(1)}} \nabla^{2} & & \\
& & \ddots & \\
& & & -\frac{1}{2 \mu_{M \bar{M}}^{(N)}} \nabla^{2}
\end{array}\right) \text {, }
$$

where $\mu_{M \bar{M}}^{(i)}$ is the reduced mass of the $i$ th meson-meson component, and matrix elements equal to zero are not displayed.

$\mathrm{V}(r)$ is the diabatic potential matrix

$$
\mathrm{V}(r)=\left(\begin{array}{cccc}
V_{\mathrm{C}}(r) & V_{\text {mix }}^{(1)}(r) & \cdots & V_{\text {mix }}^{(N)}(r) \\
V_{\text {mix }}^{(1)}(r) & T_{M \bar{M}}^{(1)} & & \\
\vdots & & \ddots & \\
V_{\text {mix }}^{(N)}(r) & & & T_{M \bar{M}}^{(N)}
\end{array}\right) .
$$

The diagonal term $V_{\mathrm{C}}(r)$ stands for the $Q \bar{Q}$ Cornell potential

$$
V_{\mathrm{C}}(r)=\sigma r-\frac{\chi}{r}+m_{Q}+m_{\bar{Q}}-\beta
$$

with $\sigma, \chi, m_{Q}$ and $\beta$ being the string tension, the color Coulomb strength, the heavy quark mass, and a constant fixing the origin of the potential respectively.

Any of the other diagonal terms stands for the mass of a meson-meson threshold

$$
T_{M \bar{M}}^{(i)}=m_{M}^{(i)}+m_{\bar{M}}^{(i)}
$$

with $m_{M}^{(i)}$ and $m_{\bar{M}}^{(i)}$ being the masses of the corresponding meson and antimeson.

The off-diagonal term $V_{\text {mix }}^{(i)}(r)$ stands for the mixing potential between the $Q \bar{Q}$ and the $i$ th meson-meson component, which can be calculated ab initio from lattice QCD.

Notice that no interaction potential between different meson-meson components is considered, what it is justified for isolated, well separated meson-meson thresholds with 
no overlap at all (including the meson widths). Notice though that an indirect interaction through their coupling to the $Q \bar{Q}$ channel is present.

Moreover, for the spherically symmetric and spinindependent diabatic potential matrix we use, each $Q \bar{Q}$ configuration with a distinct value of $\left(l_{Q \bar{Q}}, s_{Q \bar{Q}}\right)$ is taken as a channel per se, and the same for each meson-meson configuration with a distinct value of $\left(l_{M \bar{M}}^{(i)}, s_{M \bar{M}}^{(i)}\right)$. The possible values of $(l, s)$ are determined by the $J^{P C}$ quantum numbers of the quarkoniumlike system under consideration. Then, for a given $J^{P C}$ the mixing potential entering the coupled radial equations, $V_{\text {mix }}^{(i)}(r)$, is the same between any one of the $\left(l_{Q \bar{Q}}, s_{Q \bar{Q}}\right)$ and any one of the $\left(l_{M \bar{M}}^{(i)}, s_{M \bar{M}}^{(i)}\right)$ channels.

In order to calculate bound states some constraints are imposed. First, a finite number of thresholds is considered. This is justified because for a given bound state the probability of meson-meson components corresponding to thresholds far above the mass of the bound state is negligible. Additionally, only meson-meson components above the bound state mass are considered. Otherwise the free-wave behavior introduced by meson-meson components with threshold below the mass of the bound state would prevent us from obtaining a physical (bound state) solution. In practice this is done by selecting a subset of thresholds, from a given threshold up, and calculating the bound states below it. In order to avoid a possible double counting when different threshold selections are considered a one to one correspondence between the Cornell $Q \bar{Q}$ and the quarkoniumlike bound states is assumed.

Notice that a solution of the Schrödinger equation above threshold is possible, but the eigenstates would represent meson-meson scattering states. In such a context quarkoniumlike mesons would be described as resonances in a properly defined meson-meson scattering problem. A development of a diabatic description of the coupled channel meson-meson scattering, out of the scope of the present article, is under way.

The diabatic bound state approach has been applied to isoscalar $I=0$ charmoniumlike $(Q=c)$ mesons with $J^{P C}=1^{--}$and $(0,1,2)^{++}$below $4.1 \mathrm{GeV}$ for which the threshold-threshold interactions can be safely neglected. For the Cornell potential (5), standard values of the parameters

$$
\begin{gathered}
\sigma=925.6 \mathrm{MeV} / \mathrm{fm}, \\
\chi=102.6 \mathrm{MeV} \mathrm{fm}, \\
m_{c}=1840 \mathrm{MeV}, \\
\beta=855 \mathrm{MeV}
\end{gathered}
$$

have been chosen [22].
TABLE I. Low-lying open-charm meson-meson thresholds. Threshold masses $T_{M \bar{M}}^{(i)}$ from the measured charmed and charmed strange meson masses [1].

\begin{tabular}{lc}
\hline \hline$M \bar{M}$ & $T_{M \bar{M}}(\mathrm{MeV})$ \\
\hline$D \bar{D}$ & 3730 \\
$D \bar{D}^{*}(2007)$ & 3872 \\
$D_{s}^{+} D_{s}^{-}$ & 3937 \\
$D^{*}(2007) \bar{D}^{*}(2007)$ & 4014 \\
$D_{s}^{+} D_{s}^{*-}$ & 4080 \\
\hline \hline
\end{tabular}

The meson-meson thresholds, calculated from the masses of charmed and charmed strange mesons in [1], are listed in Table I. It is worth remarking that the use of the masses of the experimental thresholds introduces some implicit spin dependence in the description.

The possible values of $l_{c \bar{c}}$ and $l_{M \bar{M}}^{(i)}$ contributing to a given set of quantum numbers $J^{P C}$ are shown in Table II where the common notation $D_{(s)}$ to refer to charmed as well as to charmed strange mesons is used.

As for the mixing potential, the current dearth of unquenched lattice data for charmoniumlike systems $[23,24]$ prevents its ab initio calculation. Instead, its form has been parametrized from lattice results for bottomoniumlike systems $[15,16]$. The reasons for this parametrization have been detailed in [18]. It reads

$$
\left|V_{\text {mix }}^{(i)}(r)\right|=\frac{\Delta}{2} \exp \left\{-\frac{\left(V_{\mathrm{C}}(r)-T_{M \bar{M}}^{(i)}\right)^{2}}{2 \sigma^{2} \rho^{2}}\right\}
$$

or equivalently, at distances for which $V_{\mathrm{C}}(r) \approx$ $\sigma r+m_{Q}+m_{\bar{Q}}-\beta$,

$$
\left|V_{\text {mix }}^{(i)}(r)\right| \approx \frac{\Delta}{2} \exp \left\{-\frac{\left(r-r_{\mathrm{c}}^{(i)}\right)^{2}}{2 \rho^{2}}\right\}
$$

where $\frac{\Delta}{2}$ stands for the strength and $\rho$, the width of the second Gaussian, for the radial scale of the mixing, whereas $r_{\mathrm{c}}^{(i)}$ denotes the crossing radius defined by

$$
V_{\mathrm{C}}\left(r_{\mathrm{c}}^{(i)}\right)=T_{M \bar{M}}^{(i)}
$$

TABLE II. Values of $l_{c \bar{c}}$ and $l_{M \bar{M}}^{(i)}$ corresponding to definite values of $J^{P C}$. A missing entry for $l_{M \bar{M}}^{(i)}$ means that the particular meson-meson configuration cannot form a state with the corresponding quantum numbers.

\begin{tabular}{lcccc}
\hline \hline$J^{P C}$ & $l_{c \bar{c}}$ & $l_{D_{(s)} \bar{D}_{(s)}}$ & $l_{D_{(s)} \bar{D}_{(s)}^{*}}$ & $l_{D_{(s)}^{*} \bar{D}_{(s)}^{*}}$ \\
\hline $0^{++}$ & 1 & 0 & & 0,2 \\
$1^{++}$ & 1 & & 0,2 & 2 \\
$2^{++}$ & 1,3 & 2 & 2 & $0,2,4$ \\
$1^{--}$ & 0,2 & 1 & 1 & 1,3 \\
\hline \hline
\end{tabular}


TABLE III. Masses, $c \bar{c}$ probabilities, and meson-meson probabilities, for $(0,1,2)^{++}$and $1^{--}$charmoniumlike states calculated neglecting interaction with open thresholds. The $c \bar{c}$ probabilities from different values of $l_{c \bar{c}}$ (see Table II) have been reported inside parentheses. A missing entry under a meson-meson configuration means that the corresponding component gives negligible (i.e., probability inferior to $1 \%$ ) or no contribution at all to the state.

\begin{tabular}{|c|c|c|c|c|c|c|c|c|}
\hline$J^{P C}$ & Mass $(\mathrm{MeV})$ & $c \bar{c}$ & $D \bar{D}$ & $D \bar{D}^{*}$ & $D_{s} \bar{D}_{s}$ & $D^{*} \bar{D}^{*}$ & $D_{s} \bar{D}_{s}^{*}$ & $D_{s}^{*} \bar{D}_{s}^{*}$ \\
\hline \multirow[t]{2}{*}{$\overline{0^{++}}$} & 3508.8 & $99 \%$ & $1 \%$ & & & & & \\
\hline & 3918.9 & $60 \%$ & & & $35 \%$ & $5 \%$ & & \\
\hline \multirow[t]{2}{*}{$1^{++}$} & 3509.8 & $100 \%$ & & & & & & \\
\hline & 3871.5 & $5 \%$ & & $95 \%$ & & & & \\
\hline \multirow[t]{3}{*}{$2^{++}$} & 3508.7 & $(100,0) \%$ & & & & & & \\
\hline & 3909.0 & $(69,8) \%$ & & & $7 \%$ & $13 \%$ & $1 \%$ & $2 \%$ \\
\hline & 4006.6 & $(18,53) \%$ & & & & $28 \%$ & $1 \%$ & \\
\hline \multirow{3}{*}{$1^{--}$} & 3082.4 & $(100,0) \%$ & & & & & & \\
\hline & 3658.8 & $(92,1) \%$ & $4 \%$ & $1 \%$ & $1 \%$ & $1 \%$ & & \\
\hline & 3785.8 & $(1,95) \%$ & & $2 \%$ & $1 \%$ & $1 \%$ & & \\
\hline
\end{tabular}

Let us realize that the mixing is only effective in an interval around $r_{\mathrm{c}}^{(i)}$ determined by the value of $\rho$. The numerical values of $\rho$ and $\Delta$,

$$
\begin{gathered}
\Delta=130 \mathrm{MeV}, \\
\rho=0.3 \mathrm{fm},
\end{gathered}
$$

were obtained in [18] by fitting the mass of $\chi_{c 1}$ (3872) via a calculation involving only the $D \bar{D}^{*}$ threshold. In the present calculation we have also included the tiny effect of the $D^{*} \bar{D}^{*}$ threshold, therefore the use of the same values of the parameters gives rise to a very little discrepancy between the calculated mass of $\chi_{c 1}(3872)$ and its measured value.

The calculated spectrum of $(0,1,2)^{++}$states, containing one $c \bar{c}$ component with $l_{c \bar{c}}=1(1 p)$ or two $c \bar{c}$ components with $l_{c \bar{c}}=1,3(2 p$ and $1 f)$ and $1^{--}$states, containing one $c \bar{c}$ component with $l_{c \bar{c}}=0(1 s)$ or two $c \bar{c}$ components with $l_{c \bar{c}}=0,2(2 s$ and $1 d)$ is shown in Table III.

Let us note that the content of Table III differs from the one of Tables V and VI in [18] due to some technical improvement in the numerical calculation, to the inclusion of $l_{c \bar{c}}=3$ and $l_{M \bar{M}}^{(i)}=4$ channels which allows for the prediction of a new $2^{++}$resonance (see below), and to the exclusion of the $1^{--}$excited state assigned to $\psi(4040)$. The reason for this exclusion is that this state is peculiar in the sense that the improved calculation puts the state below the $D^{*} \bar{D}^{*}$ threshold preventing the decay to any $D^{*} \bar{D}^{*}$ channel. However, the mass correction, calculated as explained in the next section, makes the mass to be so close to the $D^{*} \bar{D}^{*}$ threshold that a small variation in the parameters (well within our expected uncertainty) puts the state above threshold permitting such decay as experimentally observed. This instability suggests that a refinement of our approximation is necessary for an appropriate description of $\psi(4040)$. This refinement may require a more complete treatment incorporating all meson-meson channels on the same foot (as in a diabatic coupled mesonmeson scattering framework not yet developed), and interactions between the bound states through their couplings to the same thresholds. It may also have to do with an improvement of the diabatic potential, through the inclusion of spin dependent terms and the consideration of a differentiated mixing for hidden strange thresholds.

For a meaningful comparison with data we have to take into account that our Cornell potential does not contain spin-dependent terms. Then, for pure $(n l) c \bar{c}$ states the calculated masses have to be compared to the $(n l)$ experimental centroids; in the other cases, where mesonmeson components are present, since they are specific for a set of $J^{P C}$ quantum numbers, the comparison has to be done with the experimental candidates with the same $J^{P C}$.

As can be checked the calculated masses for the $(0,1,2)^{++}$ground states, which are practically pure charmonium states, are pretty close to the experimental ground state centroid from $1 P_{J}$ states at $3525.30 \pm 0.11 \mathrm{MeV}$. As for the calculated masses of the first excited $(0,1,2)^{++}$ states, all containing significant meson-meson components, can be put in good correspondence with the existing experimental candidates. So, $\chi_{c 1}(3872)$, with a measured mass of $3871.69 \pm 0.17 \mathrm{MeV}$ has been assumed to correspond to the first excited $1^{++}$state, which implies that it is essentially $(95 \%)$ a $D \bar{D}^{*}$ bound state; $\chi_{c_{0}}(3860)$, with a measured mass of $3862_{-32-13}^{+26+40} \mathrm{MeV}$, corresponds to the first excited $0^{++}$state with an important $(35 \%) D_{s} \bar{D}_{s}$ component; and $\chi_{c 2}(3930)$ with a measured mass of $3922.2 \pm 1.0 \mathrm{MeV}$ corresponds to the first excited $2^{++}$ state with small but non-negligible $(7 \%) D_{s} \bar{D}_{s}$ and $(13 \%)$ $D^{*} \bar{D}^{*}$ components. It should also be remarked that the calculated masses for the first excited $(0,2)^{++}$states are in complete agreement with data regarding their positions with respect to the $D_{s} \bar{D}_{s}$ threshold, both below it.

There is an additional $\mathrm{J}^{++}$experimental resonance below $4.1 \mathrm{GeV}$, the $X(3915)$, with a measured mass of $3918.4 \pm 1.9 \mathrm{MeV}$, whose quantum numbers are not well 
established being possible a $0^{++}$as well as a $2^{++}$assignment. In [18] we tentatively identified it with $\chi_{c_{0}}(3860)$ but observed through the $(J / \psi \omega)$ decay channel. However, a $2^{++}$identification with $\chi_{c 2}(3930)$ is also feasible. In this regard, the quantitative analysis of the decay widths to open-flavor meson-meson channels may shed some light on its correct assignment.

There is also an additional theoretically predicted resonance corresponding to the second $2^{++}$excited state with no existing experimental candidate to be compared with. This prediction is quite robust in the sense that it comes out from the presence of the $1 f$ Cornell state at $4034 \mathrm{MeV}$. Hence, the experimental finding of this resonance would provide a strong support to our treatment. In this respect, the analysis of its dominant strong decay widths that we carry out later on may guide experimental searches.

As for $1^{--}$a good correspondence of the calculated masses with data can be established as well. So, the ground state is a conventional $(100 \%) c \bar{c}$ charmonium $1 s$ state, its calculated mass being pretty close to the experimental $1 \mathrm{~s}$ centroid at $3068.65 \pm 0.13 \mathrm{MeV}$ [from $J / \psi$ and $\eta_{c}(1 s)$ ].

For the first excited state, containing a very big (92\%) $2 s$ $c \bar{c}$ component and a quite small (4\%) $D \bar{D}$ component, the calculated mass is close to the measured mass of $\psi(2 s)$ : $3686.097 \pm 0.010 \mathrm{MeV}$. The second excited state is very dominantly a $1 d c \bar{c}$ state (95\%), its calculated mass comparing well with the mass of $\psi(3770)$ : $3778.1 \pm$ $0.7 \mathrm{MeV}$.

It is worth pointing out that we expect the calculated wave functions and masses for states with a nonvanishing $D_{s} \bar{D}_{s}$ and $D_{s} \bar{D}_{s}^{*}$ probability to be more uncertain that the ones calculated for states with no such components. The reason is that the parameters of the mixing potential have been fixed from the $D \bar{D}^{*}$ threshold [through the fitting of the mass of $\left.\chi_{c 1}(3872)\right]$. Then, although the use of the same parameters for the $D \bar{D}$ and $D^{*} \bar{D}^{*}$ thresholds, differing only from $D \bar{D}^{*}$ in their spins, is justified in our spin independent treatment, it may not be the case for $D_{s} \bar{D}_{s}$ and $D_{s} \bar{D}_{s}^{*}$. For these thresholds the interaction with $c \bar{c}$ involves the creation of a strange quark-antiquark pair so that the strength and radial scale for the mixing may differ from the one associated to the light quark $(u, d)$ pair creation involved in the $D \bar{D}^{*}-c \bar{c}$ interaction. Unfortunately, the lack of detailed data clearly indicating the presence of $D_{s} \bar{D}_{s}$ and $D_{s} \bar{D}_{s}^{*}$ components in some experimental resonance prevents us from fixing the specific mixing parameters for these thresholds and from estimating quantitatively the uncertainty deriving from using the same parameters as for the other thresholds. Notwithstanding this some qualitative prescription can be formulated in the sense that the bound state masses and wave functions are better described if not involving $D_{s} \bar{D}_{s}$ and $D_{s} \bar{D}_{s}^{*}$ components or involving a very small percentage of them.

Keeping this in mind, the reasonable agreement of the calculated masses with existing data for all the excited states suggests that the diabatic bound state approximation to charmoniumlike mesons, below and above threshold, may be appropriate and constitutes a good starting point for the evaluation of mass corrections and widths. Next we center on the mass shifts and widths having to do with the neglected thresholds. As we shall prove they cannot be calculated using perturbation theory against our previous assumption in [18].

\section{MASS CORRECTIONS AND WIDTHS}

In order to evaluate the effect of the $n$ neglected thresholds in the calculation of bound states with masses above these thresholds, let us start from the Hamiltonian,

$$
H_{0}=K+V_{0} \text {, }
$$

whose matrix representation in configuration space is given by the sum of $\mathrm{K}$ from (3) and

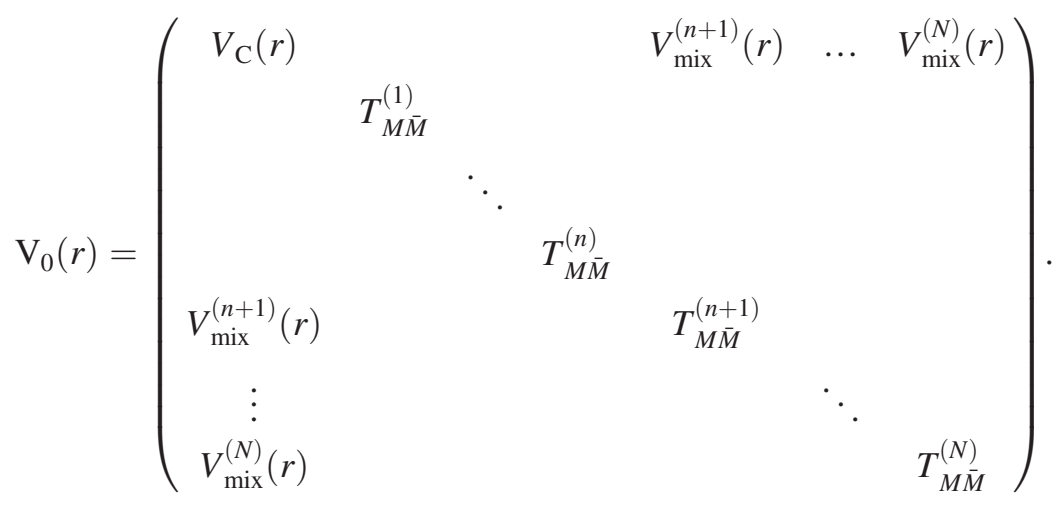


The wave functions, corresponding to solutions of the Schrödinger equation

$$
H_{0}\left|\Psi_{0}\right\rangle=E_{0}\left|\Psi_{0}\right\rangle
$$

can be expressed as

$$
\Psi_{0}(\boldsymbol{r})=\left(\begin{array}{c}
\psi_{Q \bar{Q}}(\boldsymbol{r}) \\
\tilde{\psi}_{M \bar{M}}^{(1)}(\boldsymbol{r}) \\
\vdots \\
\tilde{\psi}_{M \bar{M}}^{(n)}(\boldsymbol{r}) \\
\psi_{M \bar{M}}^{(n+1)}(\boldsymbol{r}) \\
\vdots \\
\psi_{M \bar{M}}^{(N)}(\boldsymbol{r})
\end{array}\right),
$$

where we have used the notation $\tilde{\psi}_{M \bar{M}}$ to indicate the noncoupled (free) meson-meson components.

Let us realize that any bound state obtained when neglecting the thresholds with $j=1, \ldots, n$ corresponds to a solution of (14) of the type

$$
\Psi_{\text {bound }(N-n)}^{(k)}(\boldsymbol{r})=\left(\begin{array}{c}
\psi_{Q \bar{Q}}^{(k)}(\boldsymbol{r}) \\
\\
\\
\psi_{M \bar{M}}^{(n+1)(k)}(\boldsymbol{r}) \\
\vdots \\
\psi_{M \bar{M}}^{(N)(k)}(\boldsymbol{r})
\end{array}\right)
$$

with eigenvalue $M_{(N-n)}^{(k)}$ given by the calculated bound state mass, where $k$ stands for a set of quantum numbers labeling the bound state.

On the other hand the free meson-meson states for any of the meson-meson pairs in the channels $1, \ldots, n$ correspond to solutions of (14) of the type

$$
\Psi_{\text {free }(j)}^{(\boldsymbol{p}, s)}(\boldsymbol{r})=\left(\tilde{\psi}_{M \bar{M}}^{(j)(\boldsymbol{p}, s)}(\boldsymbol{r})\right)
$$

with eigenvalues $E_{(j)}^{(\boldsymbol{p})}=T_{M \bar{M}}^{(j)}+\frac{p^{2}}{2 \mu_{M \bar{M}}^{(j)}}$, with $\boldsymbol{p}$ the relative meson-meson momentum and $s$ the total meson-meson spin. Notice that there is a continuum of eigenstates for each value of $j$.
The interaction Hamiltonian between bound and free states is provided by those mixing terms in the diabatic potential matrix $V$ that have been discarded in the construction of $V_{0}$ :

$$
H_{I} \equiv V-V_{0}
$$

Its matrix representation in configuration space reads

$$
\mathrm{H}_{I}(r)=\left(\begin{array}{cccc} 
& V_{\text {mix }}^{(1)}(r) & \cdots & V_{\text {mix }}^{(n)}(r) \\
V_{\text {mix }}^{(1)}(r) & & & \\
\vdots & & & \\
V_{\text {mix }}^{(n)}(r) & & &
\end{array}\right),
$$

where it can be seen that $H_{I}$ couples the $Q \bar{Q}$ component $\psi_{Q \bar{Q}}(\boldsymbol{r})$ of the bound state and the free meson-meson channels $\tilde{\psi}_{M \bar{M}}^{(j)}(\boldsymbol{r})$ with $j=1, \ldots, n$.

In order to study the effect of $H_{I}$ we have to realize that the exact coincidence of the energy (mass) of the bound state with one energy of the continuum for any of the $M^{(j)} \bar{M}^{(j)}$ components makes the ordinary perturbation theory inadequate (see the Appendix for a detailed explanation). Instead, a procedure based on the solution of the Schrödinger equation for $H_{0}+H_{I}$ has been developed in atomic and nuclear physics, see for instance [25], and in hadronic spectroscopy [26].

In general, for a given set of quantum numbers $J^{P C}$, this procedure involves the diagonalization of a matrix containing the interactions between bound states induced through their coupling to the same meson-meson states. However, as detailed in what follows the bound states we start from have been calculated from Hamiltonians involving different sets of neglected thresholds. Hence we can only do a consistent calculation if the meson-meson channel induced interactions between different bound states are not taken into account. Then, the problem gets reduced to the calculation of the corrections to one bound state due to its interaction with the specific neglected thresholds.

To be more precise let us consider $Q=c$. For $J^{P C}=$ $0^{++}$there is one bound (ground) state when no thresholds are neglected and two bound states (ground and first excited) when the threshold $D \bar{D}$ is neglected. The ground state is $100 \% c \bar{c}$ in both cases which means that it is decoupled from any threshold. Hence, we restrict our study to one $0^{++}$(first excited) bound state interacting with the $D \bar{D}$ threshold. Similarly, for the first excited $2^{++}$state obtained when neglecting the $D \bar{D}$ and $D \bar{D}^{*}$ thresholds, we 
calculate the corrections to the $2^{++}$(first excited) bound state interacting with $D \bar{D}$ and $D \bar{D}^{*}$. As for the second excited $2^{++}$state, obtained when neglecting the $D \bar{D}, D \bar{D}^{*}$ and $D_{s} \bar{D}_{s}$ thresholds, we calculate its corrections due to these thresholds forgetting about its interaction, induced through its coupling to $D \bar{D}$, with the first excited bound state obtained with the same Hamiltonian (notice that this first excited bound state differs from the previous one calculated without neglecting $D_{s} \bar{D}_{s}$ ).

For the $1^{--}$states we follow exactly the same protocol. So, for the second excited state we calculate its corrections due to $D \bar{D}$.

Having reduced the calculation of the corrections to the one bound state case, the physical effect of the coupling to the continuum is to dilute the bound state through a band of stationary scattering states. The expressions for the mass and width of the resulting resonance can be found in $[25,26]$. Let us detail them for one bound state and one threshold. Let $|A\rangle$ be the bound state with quantum numbers $J^{P C}$, and $|B C\rangle$ a free meson-meson state. In the center of mass frame the momentum of the resonance vanishes and

$$
\begin{aligned}
& E_{B}=m_{B}+\frac{p_{B C}^{2}}{2 m_{B}} \\
& E_{C}=m_{C}+\frac{p_{B C}^{2}}{2 m_{C}}
\end{aligned}
$$

with

$$
p_{B C}^{2}=2 \mu_{B C}\left(E_{B C}-m_{B}-m_{C}\right)
$$

and $E_{B C} \equiv E_{B}+E_{C}$.

Then the mass correction is written as

$$
\mathrm{M}-\mathrm{M}_{A}=\sum_{\substack{m_{s_{B}}, m_{s_{C}}}} \mathcal{P} \int \mathrm{d} \boldsymbol{p}_{B C} \frac{\left|\left\langle\boldsymbol{p}_{B C} ; s_{B}, m_{s_{B}}, s_{C}, m_{s_{C}}\left|H_{I}\right| A\right\rangle\right|^{2}}{\mathrm{M}-E_{B C}},
$$

where $\mathrm{M}$ is the mass of the resonance, $H_{I}$ stands for the interaction Hamiltonian operator, and $\mathcal{P} \int$ indicates the Cauchy principal value integral.

To calculate the matrix element in the numerator we manipulate it as

$$
\begin{aligned}
& \left\langle\boldsymbol{p}_{B C} ; s_{B}, m_{s_{B}}, s_{C}, m_{s_{C}}\left|H_{I}\right| A\right\rangle \\
& \quad=\sum_{s, m_{s}} C_{s_{B}, s_{C}, s}^{m_{s_{B}}, m_{s_{C}}, m_{s}}\left\langle\boldsymbol{p}_{B C} ; s_{B}, s_{C}, s, m_{s}\left|\mathrm{H}_{I}\right| A\right\rangle .
\end{aligned}
$$

The wave function for the meson-meson state reads

$$
\begin{aligned}
& \left\langle\boldsymbol{p}_{B C} ; s_{B}, s_{C}, s, m_{s} \mid \boldsymbol{r}\right\rangle \\
& =\frac{e^{-i \boldsymbol{p}_{B C} \cdot \boldsymbol{r}}}{(2 \pi)^{\frac{3}{2}}} \xi_{s}^{m_{s} \dagger} \\
& =\sum_{l, m_{l}} \sqrt{\frac{2}{\pi}} i^{-l} j_{l}\left(p_{B C} r\right) Y_{l}^{m_{l}}\left(\hat{\boldsymbol{p}}_{B C}\right) Y_{l}^{m_{l} *}(\hat{\boldsymbol{r}}) \xi_{s}^{m_{s} \dagger} \\
& =\sum_{l, m_{l}} \sqrt{\frac{2}{\pi}} i^{-l} j_{l}\left(p_{B C} r\right) Y_{l}^{m_{l}}\left(\hat{\boldsymbol{p}}_{B C}\right) \\
& \quad \times \sum_{J^{\prime}, m_{J}^{\prime}} C_{l, s, J^{\prime}}^{m_{l}, m_{s}, m_{J}^{\prime}} \mathcal{Y}_{l, s}^{J^{\prime}, m_{J}^{\prime} \dagger}(\hat{\boldsymbol{r}}),
\end{aligned}
$$

where we have defined

$$
\mathcal{Y}_{l, s}^{J^{\prime}, m_{J}^{\prime}}(\hat{\boldsymbol{r}})=\sum_{m_{l}^{\prime}, m_{s}^{\prime}} C_{l, s, J}^{m_{l}^{\prime}, m_{s}^{\prime}, m_{J}^{\prime}} Y_{l}^{m_{l}^{\prime}}(\hat{\boldsymbol{r}}) \xi_{s}^{m_{s}^{\prime}}
$$

Then as the interaction Hamiltonian only connects the $Q \bar{Q}$ component $\psi_{Q \bar{Q}}(\boldsymbol{r})$ of the bound state $A$ and the free $B C$ state, both with the same $J, m_{J}$ quantum numbers, we get

$$
\begin{aligned}
& \left\langle\boldsymbol{p}_{B C} ; s_{B}, s_{C}, s, m_{s}\left|H_{I}\right| A\right\rangle \\
& \quad=\sum_{l, m_{l}} C_{l, s, J}^{m_{l}, m_{s}, m_{J}} Y_{l}^{m_{l}}\left(\hat{\boldsymbol{p}}_{B C}\right) \mathcal{I}_{l}\left(p_{B C}\right),
\end{aligned}
$$

where we have defined

$\mathcal{I}_{l}\left(p_{B C}\right) \equiv \sqrt{\frac{2}{\pi}} i^{-l} \int \mathrm{d} r r^{2} j_{l}\left(p_{B C} r\right) V_{\text {mix }}^{(B C)}(r) u_{Q \bar{Q}}(r)$

with $u_{Q \bar{Q}}$ the sum of the $Q \bar{Q}$ radial wave functions for the several $\left(l_{Q \bar{Q}}, s_{Q \bar{Q}}\right)$ channels in the bound state $A$.

Hence we obtain

$$
\begin{aligned}
& \left\langle\boldsymbol{p}_{B C} ; s_{B}, m_{s_{B}}, s_{C}, m_{s_{C}}\left|H_{I}\right| A\right\rangle \\
& \quad=\sum_{s, m_{s}, l, m_{l}} C_{S_{B}, s_{C}, S}^{m_{s_{B}}, m_{s_{C}}, m_{s}} C_{l, s, J}^{m_{l}, m_{s}, m_{J}} Y_{l}^{m_{l}}\left(\hat{\boldsymbol{p}}_{B C}\right) \mathcal{I}_{l}\left(p_{B C}\right) .
\end{aligned}
$$

By substituting this in (22), integrating in spherical coordinates, and using angular momentum algebra it is easy to show that

$$
\mathrm{M}-\mathrm{M}_{A}=\mathcal{P} \int \mathrm{d} p_{B C} \frac{p_{B C}^{2}}{\mathrm{M}-E_{B C}} \sum_{l, s}\left|\mathcal{I}_{l}\left(p_{B C}\right)\right|^{2} .
$$

We can go further integrating over $E_{B C}$ instead of over $p_{B C}$ using

$$
\frac{\mathrm{d} E_{B C}}{\mathrm{~d} p_{B C}}=\frac{p_{B C}}{m_{B}}+\frac{p_{B C}}{m_{C}}=\frac{p_{B C}}{\mu_{B C}},
$$

which gives 


$$
p_{B C} \mathrm{~d} p_{B C}=\mu_{B C} \mathrm{~d} E_{B C}
$$

Thus we obtain

$$
\mathrm{M}-\mathrm{M}_{A}=\mathcal{P} \int \mathrm{d} E_{B C} \mu_{B C} \frac{p_{B C}}{\mathrm{M}-E_{B C}} \sum_{l, s}\left|\mathcal{I}_{l}\left(p_{B C}\right)\right|^{2},
$$

where

$$
p_{B C}=\sqrt{2 \mu_{B C}\left(E_{B C}-m_{B}-m_{C}\right)}
$$

and the sum runs over the possible values $l$ and $s$ of $B C$ that couple to $J^{P C}$. On the other hand the expression of the width reads

$$
\frac{\Gamma}{2}=\left.\pi \mu_{B C} p_{B C} \sum_{l, s}\left|\mathcal{I}_{l}(p)_{B C}\right|^{2}\right|_{E_{B C}=\mathrm{M}} .
$$

The generalization of these expressions to the case of one bound state $A$ and $n$ thresholds with masses below $\mathrm{M}_{A}$ can be done straightforwardly. So

$$
\mathrm{M}-\mathrm{M}_{A}=\sum_{j, l_{(j)}, S_{(j)}} \mathcal{P} \int \mathrm{d} E_{(j)} \mu_{B C} \frac{p_{(j)}}{\mathrm{M}-E_{(j)}}\left|\mathcal{I}_{l_{(j)}}\left(p_{(j)}\right)\right|^{2}
$$

and

$$
\frac{\Gamma}{2}=\left.\sum_{j, l_{(j)}, s_{(j)}} \pi p_{(j)}\left|\mathcal{I}_{l_{(j)}}\left(p_{(j)}\right)\right|^{2}\right|_{E_{(j)}=\mathrm{M}} .
$$

It is important to emphasize that the above expressions are beyond a perturbative treatment of $H_{I}$, see the Appendix, although the mass corrections and widths obtained are quantitatively similar to those from second order perturbation theory. Indeed, the second order perturbative correction to the energy reads

$$
\delta \mathrm{M}_{\mathrm{pert}}^{(k)}=\sum_{j, s} \int \mathrm{d} \boldsymbol{p} \frac{\left|\left\langle\Psi_{\mathrm{free}(j)}^{(\boldsymbol{p}, s)}\left|H_{I}\right| \Psi_{\mathrm{bound}(N-n)}^{(k)}\right\rangle\right|^{2}}{\mathbf{M}_{(N-n)}^{(k)}-E_{(j)}^{(\boldsymbol{p})}} .
$$

Each term in the sum over $j$ can be integrated separating the principal value and an imaginary contribution from the pole. Then the principal values and the pole contributions correspond respectively to the rhs of (35) and (36) with M substituted by $\mathbf{M}_{A}=\mathbf{M}_{(N-n)}^{(k)}$. As in practice the difference induced by this substitution is quite small the second order perturbative results yield a quite good approximation. However, as shown in the Appendix, the perturbative correction at fourth order diverges, which invalidates the perturbative treatment.

\section{RESULTS}

It is important to emphasize that our calculation of mass corrections and widths is based on the very same form of the interaction Hamiltonian we used to get the bound states. No new parameters have been introduced. Hence, the comparison to data may allow us to know the level of accuracy of our description and to think about possible improvements.

\section{A. Masses}

The calculated mass shifts and corrected masses for the $J^{P C}=1^{--}$and $(0,1,2)^{++}$states below $4.1 \mathrm{GeV}$ [except the third $1^{--}$excited state corresponding to $\left.\psi(4040)\right]$ are presented in Table IV.

Notice that we have included for completeness the first $1^{++}$excited state that we use for fixing the parameters of the mixing potential. We have not listed data from $X(3915)$ since its assignment to $0^{++}$or $2^{++}$is not well established. We shall come back to this state later on.

A look at Table IV shows that further mass corrections have to be implemented for an accurate description of data. In this regard, we have first checked that the substitution of the nonrelativistic expression of $p_{(j)}$ by the relativistic one does not make any difference. Next we analyze whether corrections to the Cornell potential could help to solve, at least in part, the observed discrepancies.

To evaluate these corrections we recall that spin splittings from a single channel Cornell potential model based on (5) and (7) have been calculated [27]. Hence, for states with approximately a $100 \% c \bar{c}$ probability we can just translate the results from [27]: $32.4 \mathrm{MeV}$ for the $1^{--}$ ground state, $-94.1 \mathrm{MeV}$ for the $0^{++}$ground state, $-29.4 \mathrm{MeV}$ for the $1^{++}$ground state, and $36.5 \mathrm{MeV}$ for the $2^{++}$ground state. This gives total masses for all these ground states differing at most $30 \mathrm{MeV}$ from the experimental ones.

TABLE IV. Calculated mass corrections, $\mathrm{M}-\mathbf{M}_{A}$, and total masses, $\mathrm{M}$, for $J^{P C}=1^{--}$and $(0,1,2)^{++}$states below $4.1 \mathrm{GeV}$. Measured masses $\mathbf{M}^{\text {Expt }}$ from [1], corresponding to the meson assignment in the last column, are also given for comparison.

\begin{tabular}{ccccc}
\hline \hline$J^{P C}$ & $\mathrm{M}-\mathrm{M}_{A}(\mathrm{MeV})$ & $\mathrm{M}(\mathrm{MeV})$ & $\mathrm{M}^{\text {Expt }}(\mathrm{MeV})$ & Meson \\
\hline $0^{++}$ & 0 & 3508.8 & $3414.71 \pm 0.3$ & $\chi_{c 0}(1 P)$ \\
& 2.0 & 3920.9 & $3862_{+26+40}^{-32-13}$ & $\chi_{c 0}(3860)$ \\
$1^{++}$ & 0 & 3509.8 & $3510.67 \pm 0.05$ & $\chi_{c 1}(1 P)$ \\
& 0 & 3871.5 & $3871.69 \pm 0.17$ & $\chi_{c 1}(3872)$ \\
$2^{++}$ & 0 & 3508.7 & $3556.17 \pm 0.07$ & $\chi_{c 2}(1 P)$ \\
& -27.9 & 3881.1 & $3922.2 \pm 1.0$ & $\chi_{c 2}(3930)$ \\
& -2.7 & 4003.9 & & \\
$1^{--}$ & 0 & 3082.4 & $3096.900 \pm 0.006$ & $J / \psi(1 S)$ \\
& 0 & 3658.8 & $3686.097 \pm 0.010$ & $\psi(2 S)$ \\
& -14.1 & 3771.7 & $3778.1 \pm 0.7$ & $\psi(3770)$ \\
\hline \hline
\end{tabular}


For the excited states, with significant meson-meson components, we cannot perform the same kind of analysis. Instead, spin dependent terms from the Cornell potential as well as spin dependent terms of the same order from the mixing and meson-meson potentials should be incorporated to the Schrödinger equation. As this is not an affordable task at present (we do not have any information on the spin dependence of the mixing potential) we just point out that the reasonable values of the masses we get for the excited states, as compared to the ones from experimental candidates, and the values of the spin splittings from the single channel Cornell potential model [27,28] suggest that the spin corrections to the masses, apart from the ones related to the use of the experimental meson masses, should be at most of a few tens of MeV. This makes us confident of our prediction of a second excited $2^{++}$state with a mass about $4 \mathrm{GeV}$.

\section{B. Widths}

The calculated widths for $J^{P C}=1^{--}$and $(0,1,2)^{++}$ states below $4.1 \mathrm{GeV}$ and above the $D \bar{D}$ threshold [except the third $1^{--}$excited state corresponding to $\left.\psi(4040)\right]$ are compiled and compared to existing data in Table V.

A look at it shows that the results for the total widths are fairly good. So, for $\psi(3770)$ the calculated $\Gamma_{D \bar{D}}$ is about a $10 \%$ below the experimental interval (we have used that $\frac{\Gamma_{D \bar{D}}^{\mathrm{Expt}}}{\Gamma_{\text {total }}^{\mathrm{Expl}}}=0.93_{-9}^{+8}$ for a better comparison), and for $\chi_{c 2}(3930)$ about a $30 \%$ above data [for $\chi_{c 0}(3860)$ better data are needed for a sensible comparison]. Moreover, we predict for $\chi_{c 2}(3930)$ a dominant decay to $D \bar{D}$, as experimentally observed. We can go further since we know for $\chi_{c 2}(3930)$ the experimental ratio

$$
\frac{\Gamma_{D \bar{D}}^{\text {Expt }} \Gamma_{\gamma \gamma}^{\text {Expt }}}{\Gamma_{\text {total }}^{\text {Expt }}}=0.21 \pm 0.04 \mathrm{keV} .
$$

We can calculate this ratio from $\Gamma_{D \bar{D}}$ and $\Gamma_{\text {total }}^{\text {Theor }}$ in Table $\mathrm{V}$, and $\Gamma\left(\chi_{c 2}(3930) \rightarrow \gamma \gamma\right)$, which can be estimated from [29] as

$$
\frac{\Gamma\left(\chi_{c 2}(3930) \rightarrow \gamma \gamma\right)}{\Gamma\left(\chi_{c 2}(1 P) \rightarrow \gamma \gamma\right)} \approx \frac{\left|\left(u_{c \bar{c}}^{\prime}(0)\right)_{\chi_{c 2}(3930)}\right|^{2}}{\left|\left(u_{c \bar{c}}^{\prime}(0)\right)_{\chi_{c 2}(1 P)}\right|^{2}}=0.80,
$$

where $u_{c \bar{c}}^{\prime}(0)$ stands for the derivative of the $c \bar{c}$ radial wave function at the origin.

Then, using the measured value

$$
\Gamma\left(\chi_{c 2}(1 P) \rightarrow \gamma \gamma\right)^{\mathrm{Expt}}=0.57 \pm 0.05 \mathrm{keV}
$$

we get

$$
\frac{\Gamma_{D \bar{D}}^{\text {Theor }} \Gamma_{\gamma \gamma}^{\text {Theor }}}{\Gamma_{\text {total }}^{\text {Theor }}} \approx 0.45 \pm 0.04 \mathrm{keV}
$$

which is a factor 2 bigger than data.

These results for $\chi_{c 2}(3930)$ seem to indicate that we overestimate the probability of the $c \bar{c}$ component since a (by hand) modest decrease in it makes the total width as well as the calculated ratio to be close to data. This overestimation may have to do with the previously mentioned need of refining our approach for a correct description of $\psi(4040)$.

This refinement should also affect our predicted width for the second excited $2^{++}$state and first excited $0^{++}$state. Anyhow, we think we can safely predict in both cases widths of at most a few tens of MeV.

It is also worth commenting about the $X(3915)$. In Ref. [1] $D^{0} \bar{D}^{* 0}$ appears as a possible decay mode. If confirmed $X(3915)$ would be a $2^{++}$resonance since this decay mode is forbidden for $0^{++}$. The $2^{++}$assignment would mean its identification with $\chi_{c 2}(3930)$. This is perfectly compatible with the average measured masses, $3918.4 \pm 1.9 \mathrm{MeV}$ for $X(3915)$ and $3922.2 \pm 1.0 \mathrm{MeV}$ for $\chi_{c 2}(3930)$. Moreover, according to our theoretical description of $\chi_{c 2}(3930)$ the discovery channel for $X(3915)$, $J / \psi \omega$, would be a natural decay mode of $\chi_{c 2}(3930)$ from its $D^{*} \bar{D}^{*}$ component via quark exchange. Hence, the confirmation of the experimental observation of the $D \bar{D}^{*}$ decay mode for $X(3915)$ could definitely settle the question about its $J^{P C}$ quantum numbers and its possible identification with $\chi_{c 2}(3930)$.

To conclude this section, let us point out that the effects of open-charm meson-meson on charmonium properties has been extensively investigated in the literature, see for example [26,28,30-37]. Although our diabatic results for mass corrections and widths cannot be directly compared to the ones calculated within different frameworks, some

TABLE V. Calculated widths to open-charm meson-meson channels. Experimental total widths $\Gamma_{\text {total }}^{\text {Expt }}$ from [1], corresponding to the meson assignment in the last column, are quoted for comparison.

\begin{tabular}{lcccccccc}
\hline \hline$J^{P C}$ & $\mathrm{M}(\mathrm{MeV})$ & $\Gamma_{D \bar{D}}(\mathrm{MeV})$ & $\Gamma_{D \bar{D}^{*}}(\mathrm{MeV})$ & $\Gamma_{D_{s} \bar{D}_{s}}(\mathrm{MeV})$ & $\Gamma_{D^{*} \bar{D}^{*}}(\mathrm{MeV})$ & $\Gamma_{\text {total }}^{\text {Theor }}(\mathrm{MeV})$ & $\Gamma_{\text {total }}^{\text {Expt }}(\mathrm{MeV})$ & Meson \\
\hline $0^{++}$ & 3920.9 & 0.6 & & & & 0.6 & $201_{-67-82}^{+154+88}$ & $\chi_{c 0}(3860)$ \\
$1^{++}$ & 3871.7 & & & & 0 & $<1.2$ & $\chi_{c 1}(3872)$ \\
$2^{++}$ & 3881.1 & 49.5 & 0.4 & & & 49.9 & $35.3 \pm 2.8$ & $\chi_{c 2}(3930)$ \\
& 4003.9 & 4.8 & 6.3 & 3.5 & & 14.5 & & \\
$1^{--}$ & 3771.7 & 20.2 & & & & 20.2 & $27.2 \pm 1.0$ & $\psi(3770)$ \\
\hline \hline
\end{tabular}


comments to contrast our approach with previous ones are in order.

A major difference of our diabatic approach with respect to all these studies is the incorporation of a lattice-based form of the mixing potential. The diabatic formalism allows to connect the mixing potential with unquenched lattice QCD results of string breaking, that is the actual physical mechanism underlying the $Q \bar{Q}$-meson-meson mixing. Conversely, in $[26,28,30]$ the mixing potential was derived from the Cornell potential whereas in most of the other studies it was generated from a phenomenological ${ }^{3} P_{0}$ model, in both cases with no clear connection to QCD. In this respect, it is important to emphasize that our parametrization of the mixing potential, which is instrumental for the calculation of masses and widths, can be straightforwardly incorporated to these studies.

Another important difference with some previous studies evaluating mass corrections and widths from open-charm meson-meson loops is the complete nonperturbative character of our calculation. As a matter of fact we have shown that a perturbative treatment to the lowest order is untenable for it is divergent to the next nonvanishing order.

Its complete nonperturbative character and the incorporation of the (lattice) QCD mixing potential are the very distinctive signatures of our diabatic approach. Certainly, at its present stage, it needs refinements for an accurate description of data, but these signatures have to be present in any description of quarkoniumlike systems from QCD.

\section{SUMMARY}

In this article, the second of a series on the diabatic approach in QCD, we have completed the study of $(0,1,2)^{++}$and $1^{--}$charmoniumlike resonances below $4.1 \mathrm{GeV}$ [except $\psi(4040)$ ]. In the first article of the series we developed a bound state approximation to a charmoniumlike resonance involving the coupling of $c \bar{c}$ and meson-meson channels with thresholds above the mass of the resonance. The (diabatic) potential matrix in the resulting Schrödinger equation for the $c \bar{c}$ and mesonmeson components was obtained from an educated guess of the corresponding static energies to be calculated in lattice QCD. This is a major difference with previous quark model studies where the nondiagonal matrix elements mixing the $c \bar{c}$ and meson-meson configurations did not have a clear connection to QCD.

In this second article, we have proceeded to a nonperturbative calculation of mass corrections and widths from meson-meson thresholds below the mass of the resonance. It is worth remarking that there is not any new free parameter in this calculation. Hence, the predicted results are directly testing our description. In this regard, we have shown that the additional consideration of spin dependent terms in the potential matrix could give account of the discrepancies between the calculated masses and data.
As for the calculated total widths, they are in fair agreement with data. A more detailed comparison with the scarce available data involving partial widths shows that the discrepancies with data could be due to the need of refining our approach beyond our corrected bound state treatment for a better wave function description.

The reasonable results we get for masses and widths make us consider as quite robust the prediction of the existence of a second excited $2^{++}$state, being dominantly a $c \bar{c} f$-wave, with a mass about $4 \mathrm{GeV}$, and width of a few tens of MeV. The experimental discovery of this resonance would provide strong support to our description.

Therefore, from our analysis of $1^{--}$and $(0,1,2)^{++}$ charmoniumlike states below $4.1 \mathrm{GeV}$, we may tentatively conclude that the diabatic approach in QCD is an adequate framework for a complete treatment of conventional and unconventional charmoniumlike resonances.

\section{ACKNOWLEDGMENTS}

This work has been supported by Ministerio de Economía, Industria y Competitividad of Spain and European Regional Development Fund Grant No. FPA2016-77177-C2-1-P, by EU Horizon 2020 Grant No. 824093 (STRONG-2020) and by Ministerio de Ciencia e Innovación and Agencia Estatal de Investigación of Spain and European Regional Development Fund Grant No. PID2019-105439 GB-C21. R. B. acknowledges a FPI fellowship from Ministerio de Ciencia, Innovacíon y Universidades of Spain under Grant No. BES-2017079860 .

\section{APPENDIX: PERTURBATIVE CORRECTIONS}

Starting from the unperturbed Hamiltonian $H_{0}$ whose eigenvectors $\left|\Psi^{(0)}\right\rangle$ and eigenvalues $E^{(0)}$ are known,

$$
H_{0}\left|\Psi^{(0)}\right\rangle=E^{(0)}\left|\Psi^{(0)}\right\rangle,
$$

the perturbation equation of order $i$ reads

$$
H_{0}\left|\Psi^{(i)}\right\rangle+H_{I}\left|\Psi^{(i-1)}\right\rangle=\sum_{j=0}^{i} E^{(j)}\left|\Psi^{(i-j)}\right\rangle,
$$

where $H_{I}$ is the interaction Hamiltonian treated as a perturbation and $\left|\Psi^{(i)}\right\rangle, E^{(i)}$ are the $i$ th order perturbative corrections to the wave function and energy respectively.

Let us now focus, for the sake of simplicity, on the perturbative solution of the diabatic problem with only one meson-meson threshold. In this case the unperturbed solutions are the discrete bound states, and the continuum of meson-meson states corresponding to the threshold:

$$
H_{0}\left|\Psi_{k}^{(0)}\right\rangle=M_{k}^{(0)}\left|\Psi_{k}^{(0)}\right\rangle
$$




$$
H_{0}\left|\Psi_{p}^{(0)}\right\rangle=E_{p}^{(0)}\left|\Psi_{p}^{(0)}\right\rangle
$$

where $M_{k}^{(0)}$ is the mass of the $k$ th bound state and $E_{p}^{(0)}$ is the c.m. meson-meson energy, given as usual by

$$
E_{p}^{(0)}=\frac{p^{2}}{2 \mu}+T
$$

where $\boldsymbol{p}$ is the relative momentum, $p$ its modulus, $\mu$ the reduced mass, and $T$ the threshold mass.

We now set off to calculate the perturbative corrections $\left|\Psi_{k}^{(i)}\right\rangle$ and $M_{k}^{(i)}$ of some discrete state labeled by $k$, assuming for simplicity that the unperturbed discrete spectrum is nondegenerate.

To clearly distinguish between the unperturbed bound states and unperturbed free meson-meson states, let us introduce the notation

$$
\left|\psi_{k}\right\rangle \equiv\left|\Psi_{k}^{(0)}\right\rangle, \quad\left|\varphi_{p}\right\rangle \equiv\left|\Psi_{p}^{(0)}\right\rangle .
$$

Let us note that in perturbation theory, for $i>0$ one has $\left\langle\psi_{k} \mid \Psi_{k}^{(i)}\right\rangle=0$ by construction.

By multiplying Eq. (A2) for $i=1$ on the left by $\left\langle\psi_{k}\right|$, and taking into account that the interaction Hamiltonian only connects bound states and continuum states, it can be easily shown that $M_{k}^{(1)}=0$, i.e., the first order correction to the mass vanishes. On the other hand, by multiplying Eq. (A2) for $i=1$ on the left by $\left\langle\psi_{k^{\prime}}\right|$ with $k^{\prime} \neq k$, it is straightforward to show that $\left\langle\psi_{k^{\prime}} \mid \Psi_{k}^{(1)}\right\rangle=0$. This is, there is no first order correction to the wave function from other discrete states. Actually the only first order correction to the wave function comes from meson-meson states, and can be calculated by multiplying Eq. (A2) for $i=1$ on the left by $\left\langle\varphi_{p}\right|$ :

$$
E_{p}^{(0)}\left\langle\varphi_{\boldsymbol{p}} \mid \Psi_{k}^{(1)}\right\rangle+\left\langle\varphi_{\boldsymbol{p}}\left|H_{I}\right| \psi_{k}\right\rangle=M_{k}^{(0)}\left\langle\varphi_{\boldsymbol{p}} \mid \Psi_{k}^{(1)}\right\rangle,
$$

so that we can express

$$
\left\langle\varphi_{p} \mid \Psi_{k}^{(1)}\right\rangle=\frac{\left\langle\varphi_{p}\left|H_{I}\right| \psi_{k}\right\rangle}{M_{k}^{(0)}-E_{p}^{(0)}+i \epsilon},
$$

where as customary we have introduced an imaginary factor $i \epsilon$ to deal with the case $E_{p}^{(0)}=M_{k}^{(0)}$.

Higher order corrections are calculated exactly in the same way as the first order ones. It is sufficient to multiply the perturbation equation (A2) of order $i$ by $\left\langle\psi_{k}\right|,\left\langle\psi_{k^{\prime}}\right|$ with $k^{\prime} \neq k$, and $\left\langle\varphi_{p}\right|$ to obtain the $i$ th order mass correction and wave function corrections respectively. Thus the second order mass correction reads

$$
M_{k}^{(2)}=\left\langle\psi_{k}\left|H_{I}\right| \Psi_{k}^{(1)}\right\rangle=\int \mathrm{d} \boldsymbol{p} \frac{\left|\left\langle\varphi_{p}\left|H_{I}\right| \psi_{k}\right\rangle\right|^{2}}{M_{k}^{(0)}-E_{p}^{(0)}+i \epsilon} .
$$

This term can be physically interpreted as a one mesonmeson loop correction to the mass. Note that as $\epsilon$ goes to zero the simple pole of the integrand moves on to the real axis, at $E_{p}^{(0)}=M_{k}^{(0)}$. For states above threshold we have $M_{k}^{(0)}>T$, which causes the pole to fall within the integration range. Then for $\epsilon \rightarrow 0$ the integral splits in a real principal part corresponding to the mass shift and an imaginary contribution that is proportional to the decay width of the quarkonium state to a meson-meson pair. More precisely, it can be shown that the imaginary contribution from this one loop correction is exactly half the decay amplitude calculated at tree level, in accordance with the optical theorem.

The second order correction to the wave function is calculated from

$M_{k^{\prime}}^{(0)}\left\langle\psi_{k^{\prime}} \mid \Psi_{k}^{(2)}\right\rangle+\left\langle\psi_{k^{\prime}}\left|H_{I}\right| \Psi_{k}^{(1)}\right\rangle=M_{k}^{(0)}\left\langle\psi_{k^{\prime}} \mid \Psi_{k}^{(2)}\right\rangle$,

yielding

$$
\begin{aligned}
\left\langle\psi_{k^{\prime}} \mid \Psi_{k}^{(2)}\right\rangle & =\frac{\left\langle\psi_{k^{\prime}}\left|H_{I}\right| \Psi_{k}^{(1)}\right\rangle}{M_{k}^{(0)}-M_{k^{\prime}}^{(0)}} \\
& =\int \mathrm{d} \boldsymbol{p} \frac{\left\langle\psi_{k^{\prime}}\left|H_{I}\right| \varphi_{p}\right\rangle}{M_{k}^{(0)}-M_{k^{\prime}}^{(0)}} \frac{\left\langle\varphi_{p}\left|H_{I}\right| \psi_{k}\right\rangle}{M_{k}^{(0)}-E_{p}^{(0)}+i \epsilon},
\end{aligned}
$$

which can be physically interpreted as a mixing between quarkonium states mediated by one meson-meson loop.

At third order the mass correction vanishes, $M_{k}^{(3)}=0$, as can be easily shown. The only nonvanishing contribution, the wave function correction from the continuum states, is derived from

$$
\begin{aligned}
& E_{p}^{(0)}\left\langle\varphi_{\boldsymbol{p}} \mid \Psi_{k}^{(3)}\right\rangle+\left\langle\varphi_{\boldsymbol{p}}\left|H_{I}\right| \Psi_{k}^{(2)}\right\rangle \\
& \quad=M_{k}^{(0)}\left\langle\varphi_{\boldsymbol{p}} \mid \Psi_{k}^{(3)}\right\rangle+M_{k}^{(2)}\left\langle\varphi_{\boldsymbol{p}} \mid \Psi_{k}^{(1)}\right\rangle
\end{aligned}
$$

which gives

$$
\left\langle\varphi_{p} \mid \Psi_{k}^{(3)}\right\rangle=\frac{\left\langle\varphi_{p}\left|H_{I}\right| \Psi_{k}^{(2)}\right\rangle}{M_{k}^{(0)}-E_{p}^{(0)}+i \epsilon}-M_{k}^{(2)} \frac{\left\langle\varphi_{p} \mid \Psi_{k}^{(1)}\right\rangle}{M_{k}^{(0)}-E_{p}^{(0)}+i \epsilon} .
$$


Finally, the fourth order mass correction reads

$$
\begin{aligned}
M_{k}^{(4)}= & \left\langle\psi_{k}\left|H_{I}\right| \Psi_{k}^{(3)}\right\rangle=\sum_{k^{\prime} \neq k} \iint \mathrm{d} \boldsymbol{p} \mathrm{d} \boldsymbol{p}^{\prime} \frac{\left\langle\psi_{k}\left|H_{I}\right| \varphi_{p}\right\rangle\left\langle\varphi_{p}\left|H_{I}\right| \psi_{k^{\prime}}\right\rangle\left\langle\psi_{k^{\prime}}\left|H_{I}\right| \varphi_{p^{\prime}}\right\rangle\left\langle\varphi_{p^{\prime}}\left|H_{I}\right| \psi_{k}\right\rangle}{\left(M_{k}^{(0)}-E_{p}^{(0)}+i \epsilon\right)\left(M_{k}^{(0)}-M_{k^{\prime}}^{(0)}\right)\left(M_{k}^{(0)}-E_{p^{\prime}}^{(0)}+i \epsilon\right)} \\
& -\left(\int \mathrm{d} \boldsymbol{p}^{\prime} \frac{\left|\left\langle\varphi_{p^{\prime}}\left|H_{I}\right| \psi_{k}\right\rangle\right|^{2}}{M_{k}^{(0)}-E_{p^{\prime}}^{(0)}+i \epsilon}\right) \cdot\left(\int \mathrm{d} \boldsymbol{p} \frac{\left|\left\langle\varphi_{p}\left|H_{I}\right| \psi_{k}\right\rangle\right|^{2}}{\left(M_{k}^{(0)}-E_{p}^{(0)}+i \epsilon\right)^{2}}\right),
\end{aligned}
$$

where it can be seen that when $M_{k}^{(0)}>T$ the last integral of the second line is divergent as $\epsilon \rightarrow 0$, since the integrand has a double pole.

The divergence of the fourth order mass correction reflects the fact that the presence of continuum states with energy equal to the mass of some discrete states invalidates the perturbative treatment for these states.

[1] P. A. Zyla et al. (Particle Data Group), Review of particle physics, Prog. Theor. Exp. Phys. 2020, 083C01 (2020).

[2] N. Brambilla et al., Heavy quarkonium: Progress, puzzles, and opportunities, Eur. Phys. J. C 71, 1534 (2011).

[3] G. T. Bodwin, E. Braaten, E. Eichten, S. L. Olsen, T. K. Pedlar, and J. Russ, Quarkonium at the frontiers of high energy physics: A snowmass white paper, in Proceedings, 2013 Community Summer Study on the Future of U.S. Particle Physics: Snowmass on the Mississippi (CSS2013), Minneapolis, MN, USA, 2013, edited by N. A. Graf, M.E. Peskin, and J.L. Rosner, SLAC-econf-C130729.2, FERMILAB-CONF-13-648, SLAC-PUB-15960 (2013).

[4] H.-X. Chen, W. Chen, X. Liu, and S.-L. Zhu, The hiddencharm pentaquark and tetraquark states, Phys. Rep. 639, 1 (2016).

[5] A. Hosaka, T. Iijima, K. Miyabayashi, Y. Sakai, and S. Yasui, Exotic hadrons with heavy flavors: $\mathrm{X}, \mathrm{Y}, \mathrm{Z}$, and related states, Prog. Theor. Exp. Phys. 2016, $062 \mathrm{C} 01$ (2016).

[6] H.-X. Chen, W. Chen, X. Liu, Y.-R. Liu, and S.-L. Zhu, A review of the open charm and open bottom systems, Rep. Prog. Phys. 80, 076201 (2017).

[7] Y. Dong, A. Faessler, and V. E. Lyubovitskij, Description of heavy exotic resonances as molecular states using phenomenological Lagrangians, Prog. Part. Nucl. Phys. 94, 282 (2017).

[8] A. Esposito, A. Pilloni, and A. Polosa, Multiquark resonances, Phys. Rep. 668, 1 (2017).

[9] R. F. Lebed, R.E. Mitchell, and E. S. Swanson, Heavyquark QCD exotica, Prog. Part. Nucl. Phys. 93, 143 (2017).

[10] F.-K. Guo, C. Hanhart, U.-G. Meißner, Q. Wang, Q. Zhao, and B.-S. Zou, Hadronic molecules, Rev. Mod. Phys. 90, 015004 (2018).

[11] S. L. Olsen, T. Skwarnicki, and D. Zieminska, Nonstandard heavy mesons and baryons: Experimental evidence, Rev. Mod. Phys. 90, 015003 (2018).

[12] Y.-R. Liu, H.-X. Chen, W. Chen, X. Liu, and S.-L. Zhu, Pentaquark and tetraquark states, Prog. Part. Nucl. Phys. 107, 237 (2019).
[13] C.-Z. Yuan and S.L. Olsen, The BESIII physics programme, Nat. Rev. Phys. 1, 480 (2019).

[14] N. Brambilla, S. Eidelman, C. Hanhart, A. Nefediev, C.-P. Shen, C. E. Thomas, A. Vairo, and C.-Z. Yuan, The $X Y Z$ states: Experimental and theoretical status and perspectives, Phys. Rep. 873, 1 (2020).

[15] G. S. Bali, H. Neff, T. Düssel, T. Lippert, and K. Schilling (SESAM Collaboration), Observation of string breaking in QCD, Phys. Rev. D 71, 114513 (2005).

[16] J. Bulava, B. Hörz, F. Knechtli, V. Koch, G. Moir, C. Morningstar, and M. Peardon, String breaking by light and strange quarks in QCD, Phys. Lett. B 793, 493 (2019).

[17] P. Bicudo, M. Cardoso, N. Cardoso, and M. Wagner, Bottomonium resonances with $I=0$ from lattice QCD correlation functions with static and light quarks, Phys. Rev. D 101, 034503 (2020).

[18] R. Bruschini and P. González, Diabatic description of charmoniumlike mesons, Phys. Rev. D 102, 074002 (2020).

[19] M. Baer, Beyond Born-Oppenheimer: Electronic Nonadiabatic Coupling Terms and Conical Intersections (John Wiley \& Sons, New York, 2006).

[20] K. J. Juge, J. Kuti, and C. J. Morningstar, Ab Initio Study of Hybrid $\bar{b} g b$ Mesons, Phys. Rev. Lett. 82, 4400 (1999).

[21] E. Braaten, C. Langmack, and D. H. Smith, BornOppenheimer approximation for the $X Y Z$ mesons, Phys. Rev. D 90, 014044 (2014).

[22] E. J. Eichten and C. Quigg, Mesons with beauty and charm: Spectroscopy, Phys. Rev. D 49, 5845 (1994).

[23] G. S. Bali, S. Collins, and C. Ehmann, Charmonium spectroscopy and mixing with light quark and open charm states from $n_{F}=2$ lattice QCD, Phys. Rev. D 84, 094506 (2011).

[24] G. Bali, S. Collins, D. Mohler, M. Padmanath, S. Piemonte, S. Prelovsek, and S. Weishäupl, Charmonium resonances on the lattice, EPJ Web Conf. 175, 05020 (2018).

[25] U. Fano, Effects of configuration interaction on intensities and phase shifts, Phys. Rev. 124, 1866 (1961). 
[26] E. Eichten, K. Gottfried, T. Kinoshita, K. D. Lane, and T. M. Yan, Charmonium: The model, Phys. Rev. D 17, 3090 (1978); Erratum, Phys. Rev. D 21, 313 (1980).

[27] E. Eichten and F. Feinberg, Spin-dependent forces in quantum chromodynamics, Phys. Rev. D 23, 2724 (1981).

[28] E. J. Eichten, K. Lane, and C. Quigg, Charmonium levels near threshold and the narrow state $X(3872) \rightarrow \pi^{+} \pi^{-} J / \psi$, Phys. Rev. D 69, 094019 (2004).

[29] W. Kwong, P. B. Mackenzie, R. Rosenfeld, and J. L. Rosner, Quarkonium annihilation rates, Phys. Rev. D 37, 3210 (1988).

[30] E. J. Eichten, K. Lane, and C. Quigg, New states above charm threshold, Phys. Rev. D 73, 014014 (2006); Erratum, Phys. Rev. D 73, 079903 (2006).

[31] T. Barnes, S. Godfrey, and E. S. Swanson, Higher charmonia, Phys. Rev. D 72, 054026 (2005).

[32] T. Barnes and E. S. Swanson, Hadron loops: General theorems and application to charmonium, Phys. Rev. C 77, 055206 (2008).
[33] M. R. Pennington and D. J. Wilson, Decay channels and charmonium mass shifts, Phys. Rev. D 76, 077502 (2007).

[34] I. V. Danilkin and Y. A. Simonov, Channel coupling in heavy quarkonia: Energy levels, mixing, widths, and new states, Phys. Rev. D 81, 074027 (2010).

[35] J. Ferretti, G. Galatà, and E. Santopinto, Interpretation of the $X(3872)$ as a charmonium state plus an extra component due to the coupling to the meson-meson continuum, Phys. Rev. C 88, 015207 (2013).

[36] P. G. Ortega, J. Segovia, D. R. Entem, and F. Fernández, Charmonium resonances in the $3.9 \mathrm{GeV} / c^{2}$ energy region and the $X(3915) / X(3930)$ puzzle, Phys. Lett. B 778, 1 (2018).

[37] E. van Beveren and G. Rupp, Modern meson spectroscopy: The fundamental role of unitarity, Prog. Part. Nucl. Phys. 117, 103845 (2021). 